\title{
Hadronic effects in leptonic systems: muonium hyperfine structure and anomalous magnetic moment of muon
}

\author{
S. I. Eidelman, S. G. Karshenboim, and V. A. Shelyuto
}

\begin{abstract}
Contributions of hadronic effects to the muonium physics and anomalous magnetic moment of muon are considered. Special attention is paid to higher-order effects and the uncertainty related to the hadronic contribution to the hyperfine structure interval in the ground state of muonium.
\end{abstract}

PACS Nos.: 12.20.-m, 36.10.Dr, 31.30.Jv and 13.65.+i

Résumé : French version of abstract (supplied by CJP)

[Traduit par la rédaction]

\section{Introduction}

Quantum electrodynamics (QED) is a theory which covers all interactions of leptons (electrons and muons) with photons. However, it may be not sufficient even for pure leptonic systems that are not free of hadronic effects appearing because of hadronic intermediate states. Such effects cannot be calculated $a b$ initio and additional data on these states are needed. Since the data can be achieved mainly from experiment, their availability and uncertainty impose some principal limits on any QED calculations.

Recently a number of projects on intensive muon sources have appeared [1] and a new generation of precision experiments on muonium may appear in near future. Therefore it is timely to investigate the principal limits of QED tests related to hadronic physics. Here we discuss hadronic effects in muonium and free muon.

\section{Hadronic effects in muonium hyperfine structure: leading contribution}

The leading hadronic contribution to the hyperfine splitting of the muonium ground state comes from the diagram depicted in Fig. 1. We separate QED and hadronic effects in this diagram [2]

$\Delta E_{\mathrm{hadr}}($ leading $)=-2 \frac{\alpha^{2}}{\pi^{2}} \frac{m_{e} m_{\mu}}{m_{\mu}^{2}-m_{e}^{2}} \frac{E_{F}}{1+a_{\mu}} \int d s K_{\mathrm{Mu}}(s) \rho(s)$

Received ???. Accepted ???

S. I. Eidelman. Budker Institute for Nuclear Physics and Novosibirsk University, Novosibirsk, 630090, Russia S. G. Karshenboim. D. I. Mendeleev Institute for Metrology, St. Petersburg, 189620, Russia and Max-PlanckInstitut für Quantenoptik, Garching, 85748, Germany

V. A. Shelyuto. D. I. Mendeleev Institute for Metrology, St. Petersburg, 189620, Russia 
Fig. 1. The leading hadronic contribution to the muonium hyperfine structure

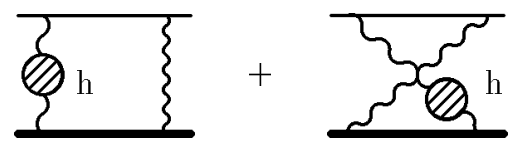

Fig. 2. The skeleton two-phonon exchange diagrams

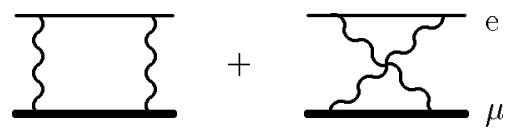

and evaluate them independently. Here $K_{\mathrm{Mu}}(s)$ is a result of the calculation of a two-photon exchange diagram with one massive photon $\left(s=\lambda^{2}\right)$, the Fermi energy

$E_{F}=\frac{8}{3}(Z \alpha)^{4} \frac{m_{e}^{2} m_{\mu}^{2}}{\left(m_{\mu}+m_{e}\right)^{3}}\left(1+a_{\mu}\right)$

is a nonrelativistic value for the hyperfine structure interval, $s$ is the center-of-mass energy squared and $\rho(s)$ is the hadronic spectral function:

$\rho(s)=\frac{2}{3 s} R(s)=\frac{\sigma\left(e^{+} e^{-} \rightarrow \gamma \rightarrow \text { hadrons }\right)}{2 \pi \alpha^{2}}$.

The Fermi energy determined above contains the anomalous magnetic moment of muon, $a_{\mu}$, which is also affected by hadronic effects (see. Sect. 4).

The basic expression has been obtained in Ref. [2]. Here we briefly reproduce the derivation. We start from the evaluation of the two-photon skeleton contribution (Fig. 2)

$\Delta E_{\mathrm{skel}}=E_{F} \frac{Z \alpha}{\pi} \frac{m_{\mu} m_{e}}{m_{\mu}^{2}-m_{e}^{2}} \int_{0}^{\infty} \frac{d k^{2}}{k^{2}}\left(J\left(k, m_{\mu}\right)-J\left(k, m_{e}\right)\right)$,

where

$J(k, \mu)=2\left[\frac{1}{k} \sqrt{k^{2}+4 \mu^{2}}-1\right]-\frac{1}{4 \mu^{2}}\left[k \sqrt{k^{2}+4 \mu^{2}}-k^{2}-2 \mu^{2}\right]$.

We introduce hadronic polarization effects with a dispersion substitute for the photon propagator

$\frac{1}{k^{2}} \rightarrow \frac{\alpha}{\pi} \int \frac{d s \rho(s)}{k^{2}+s}$.

and after $k$-integration we obtain

$$
\begin{aligned}
K_{\mathrm{Mu}}(s)= & \left(\frac{s}{4 m_{\mu}^{2}}+2\right) \sqrt{1-\frac{4 m_{\mu}^{2}}{s}} \ln \frac{1+\sqrt{1-\frac{4 m_{\mu}^{2}}{s}}}{1-\sqrt{1-\frac{4 m_{\mu}^{2}}{s}}} \\
& -\left(\frac{s}{4 m_{\mu}^{2}}+\frac{3}{2}\right) \ln \frac{s}{m_{\mu}^{2}}+\frac{1}{2}+\ldots .
\end{aligned}
$$


Fig. 3. Asymptotic behaviour of hadronic kernel. $\Delta K_{\mathrm{Mu}}(s)=K_{\mathrm{Mu}}(s)-K_{\mathrm{Mu}}^{(0)}(s)$.

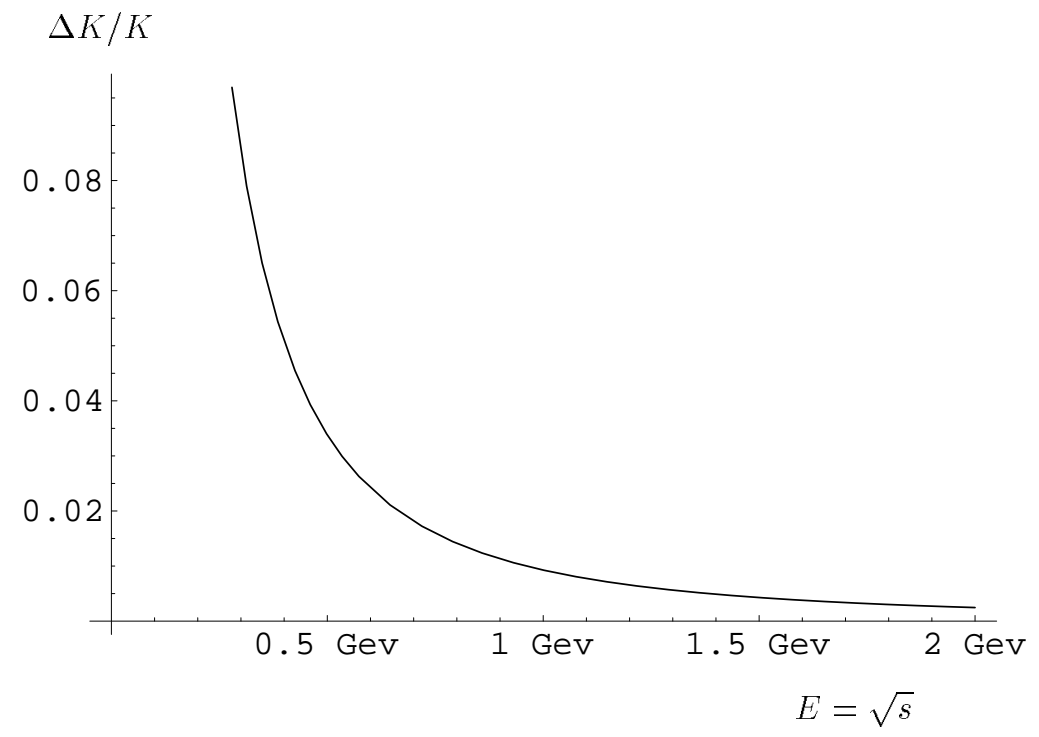

We are interested in relatively high $s$ and the expansion of the exact result

$$
\begin{aligned}
K_{\mathrm{Mu}}(s)=- & \left\{\frac{4 m_{\mu}^{2}}{s}\left[\frac{9}{8} \ln \frac{s}{m_{\mu}^{2}}+\frac{15}{16}\right]+\left(\frac{4 m_{\mu}^{2}}{s}\right)^{2}\left[\frac{5}{16} \ln \frac{s}{m_{\mu}^{2}}-\frac{17}{96}\right]\right. \\
& \left.+\left(\frac{4 m_{\mu}^{2}}{s}\right)^{3}\left[\frac{21}{128} \ln \frac{s}{m_{\mu}^{2}}-\frac{73}{512}\right]+\ldots\right\}
\end{aligned}
$$

is useful for further evaluations. For $s=m_{\rho}^{2}$ the leading term

$K_{\mathrm{Mu}}^{(0)}(s)=\frac{4 m_{\mu}^{2}}{s}\left[\frac{9}{8} \ln \frac{s}{m_{\mu}^{2}}+\frac{15}{16}\right]$

is a good enough approximation (within 2\%). For higher $s$ it is even better (see Fig. 3).

A simple model can be used to estimate the leading hadronic contribution (cf. Ref. [3]). The spectral function consists of several narrow-pole contributions $(\rho, \omega, \phi)$

$\rho_{\text {pole }}(s)=\sum_{\text {res }} \frac{4 \pi^{2}}{f_{\text {res }}^{2}} \delta\left(s-M_{\text {res }}^{2}\right)=\frac{3 \pi \Gamma\left(\text { res } \rightarrow e^{+} e^{-}\right)}{\alpha^{2} M_{\text {res }}} \delta\left(s-M_{\text {res }}^{2}\right)$

and background with $R=2$ for $1 \mathrm{Gev}^{2}<s<4 \mathrm{Gev}^{2}$ and $R=4$ for $4 \mathrm{Gev}^{2}<s$. Here $M_{\text {res }}, \Gamma$ (res $\rightarrow$ $\left.e^{+} e^{-}\right)$and $f_{\text {res }}$ are the resonance mass, leptonic width and coupling constant, respectively. The results are summarized in Table 1. We estimate a systematic uncertainty of the model as $10 \%$. That is not good enough for a calculation of the leading term but sufficient for higher-order hadronic corrections. 
Table 1. Estimation of the leading hadronic contribution to the hyperfine structure in a simplified model.

\begin{tabular}{ccc}
\hline Contribution & $\Delta E$ & $\Delta E / \Delta E_{\text {hadr }}$ \\
& {$[\mathrm{kHz}]$} & {$[\%]$} \\
\hline$\rho$ & $0.151(7)$ & $65(3)$ \\
$\omega$ & 0.013 & $5.5(2)$ \\
$\phi$ & 0.014 & $6.0(2)$ \\
$s=1-4 \mathrm{Gev}^{2}$ & 0.045 & 19 \\
$s>4 \mathrm{Gev}^{2}$ & 0.009 & 3.8 \\
\hline Total & $0.232(7)$ & $99(3)$ \\
\hline
\end{tabular}

Any realistic calculation of the leading hadronic term targeting a higher accuracy should deal with experimental data on $\mathrm{e}^{+} \mathrm{e}^{-}$annihilation into hadrons (see e.g. Refs. [4, 5, 6]). The most accurate recent result $[5]^{1}$ is

$\Delta E_{\text {hadr }}($ leading $)=0.233(3) \mathrm{kHz}$.

Since the publication of Ref. [5], some new experimental data appeared and the result can be now updated. These new data are:

- The CMD-2 detector has recently published the final results of their analysis for the reaction $e^{+} e^{-} \rightarrow \pi^{+} \pi^{-}$in the c. m. energy range 610 to $960 \mathrm{MeV}$ [7]. Because of the new, more precise approach to calculating radiative corrections which also include a correction for the final state radiation, the values of the cross section are slightly smaller than before.

- The new data on the $\phi$ meson leptonic width obtained by the CMD-2 and SND groups in Novosibirsk [8].

As a result, the updated value of the leading order hadronic contribution becomes $231.2 \pm 2.9 \mathrm{~Hz}$ compared to our last year value of $233.3 \pm 3.1 \mathrm{~Hz}$ quoted above. We expect that after the analysis of other hadronic modes is completed in Novosibirsk, the precision of the leading order contribution can be improved to about $2 \mathrm{~Hz}$.

\section{Hadronic effects in muonium hyperfine structure: next-to-leading term}

With an accuracy at a one-percent level for the leading hadronic contribution one has to take into account higher-order hadronic effects. The corresponding diagrams for some higher-order hadronic terms are presented in Fig. 4. The first evaluation presented in Ref. [2] gave a result

$\Delta E_{\text {hadr }}($ non - leading $)=0.007(2) \mathrm{kHz}$.

Most of the contributions can be expressed in terms of corrections to $K_{\mathrm{Mu}}$ in the form

$$
\begin{aligned}
\Delta K & =-\frac{\alpha}{\pi} \frac{4 m_{\mu}^{2}}{s}\left\{\ln \frac{9}{8} \frac{m_{\mu}^{2}}{m_{e}^{2}} \ln \frac{s}{m_{\mu}^{2}}+\frac{9}{8} \ln ^{2} \frac{s}{m_{\mu}^{2}}+\frac{15}{16} \ln \frac{m_{\mu}^{2}}{m_{e}^{2}}+C_{1} \ln \frac{s}{m_{\mu}^{2}}+C_{2}\right\} \\
& \simeq-\frac{\alpha}{\pi} \frac{4 m_{\mu}^{2}}{s}\left\{47.6+17.7+10.0+C_{1} \cdot 4.0+C_{2}\right\} .
\end{aligned}
$$

\footnotetext{
1 The result of Ref. [6] has been obtained using $\tau$ lepton data and the accuracy quoted there is overestimated. The discussion of the possibility to use $\tau$ data can be found in Sect. 4 .
} 
Fig. 4. The next-to-leading hadronic contribution to the hyperfine splitting in muonium
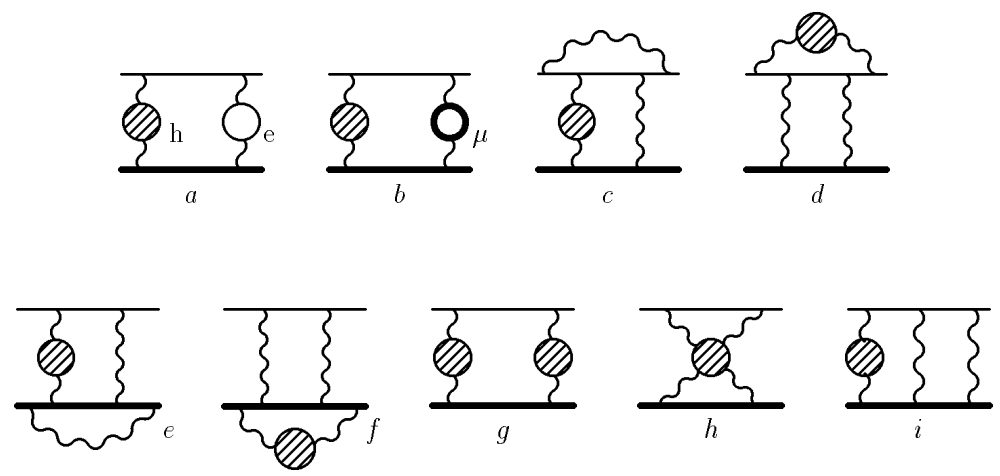

Table 2. Higher-order hadronic contributions to muonium hyperfine splitting. The results are presented in units of $\alpha / \pi \cdot E_{\text {hadr }}$ (leading).

\begin{tabular}{cc}
\hline Correction & $\begin{array}{c}\text { Contribution } \\
{\left[\alpha / \pi \cdot E_{\mathrm{hadr}}\right]}\end{array}$ \\
\hline$\Delta E_{\mathrm{a}}$ & 9.8 \\
$\Delta E_{\mathrm{b}}$ & $1.6(7)$ \\
$\Delta E_{\mathrm{c}}$ & $-1.0(3)$ \\
$\Delta E_{\mathrm{d}}$ & \pm 0.1 \\
$\Delta E_{\mathrm{e}}$ & $-1.0(3)$ \\
$\Delta E_{\mathrm{f}}$ & \pm 0.1 \\
$\Delta E_{\mathrm{g}}$ & $0.10(2)$ \\
$\Delta E_{\mathrm{h}}$ & \pm 0.1 \\
$\Delta E_{\mathrm{i}}$ & \pm 0.3 \\
\hline$\Delta E_{\mathrm{high}}$ & $9.2(14)$ \\
\hline
\end{tabular}

The numerical values are given for $s=m_{\rho}^{2}$. We present here the calculation of double-logarithmic terms and that of a single-logarithmic term with the bigger logarithm $\ln \left(m_{\mu}^{2} / m_{e}^{2}\right) \simeq 11>\ln \left(m_{\rho}^{2} / m_{\mu}^{2}\right) \simeq$ 4. A calculation of other logarithmic contributions is in progress.

The biggest contributions come from Fig. $4 a$. The result is

$\Delta K_{\mathrm{a}}=-3 \frac{\alpha}{3 \pi} \frac{4 m_{\mu}^{2}}{s}\left\{\left[\frac{9}{16} \ln ^{2} \frac{s}{m_{\mu}^{2}}-\frac{3 \pi^{2}}{8}-\frac{27}{32}\right]+\left[\frac{9}{8} \ln \frac{s}{m_{\mu}^{2}}+\frac{15}{16}\right]\left[\ln \frac{m_{\mu}^{2}}{m^{2}}-\frac{5}{3}\right]\right\}$.

Our final estimate is

$\Delta E_{\text {hadr }}($ non - leading $)=0.005(2) \mathrm{kHz}$.

The contributions are summarized in Table 2. The result is somewhat lower than in Ref. [2] mainly because of single-logarithmic contributions from Fig. $4 c$ and $e$.

Recently higher-order hadronic effects were also studied in Ref. [9]. In particular, the hadronic light-by-light contributions were under examination. The contribution of the diagram in Fig. $4 h$ is $1.5 \mathrm{mHz}$ and so it is consistent with our conservative estimation. A bigger contribution of $3.65 \mathrm{~Hz}$ 
Fig. 5. Leading hadronic contribution to the anomalous magnetic moment of muon

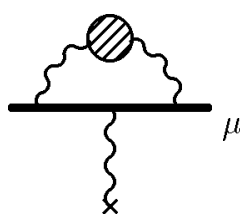

found there (related to Fig. 2 of Ref. [9]) is actually due to the correction to the anomalous magnetic moment of muon and affects hyperfine splitting accordingly to Eq. (2). Therefore it should be excluded from our consideration.

More accurate calculations are in progress and we hope to reduce the uncertainty by a factor of 4 [10].

\section{Anomalous magnetic moment of muon}

Since the 2001 publication of the E821 result on the anomalous magnetic moment of muon [11], a lot of efforts have been put on the new estimation of the hadronic correction to the muon anomaly, $a_{\mu}^{\text {had }}$, see e.g. Ref. [12]. However, these works were based on either not final experimental data or involved various theoretical assumptions. In view of the extreme importance of the E821 result and possible indications to deviation from the Standard Model it is necessary to have a model-independent analysis based exclusively on the data, similar to that of Ref. [13]. The new calculation based on the most recent data from $e^{+} e^{-}$annihilation at low energy in Novosibirsk and Beijing as well as from $\tau$ decay data, which also includes new developments in theory, has recently been published [14]. It showed a real breakthrough in the accuracy of the $a_{\mu}^{\text {had }}$ estimation which became possible after the final data on $e^{+} e^{-} \rightarrow \pi^{+} \pi^{-}$from CMD-2 with a $0.6 \%$ systematic uncertainty have been published [7] and, in addition, the final analysis of the R measurement by BES between 2 and $5 \mathrm{GeV}$ appeared [15]. The resulting accuracy of the estimate increases dramatically and reaches $\simeq 70 \cdot 10^{-11}$ based on $e^{+} e-$ data only. This accuracy is much better compared to the uncertainty of $153 \cdot 10^{-11}$ obtained in 1995 in Ref. [13] and is very close to that obtained in the 1998 analysis of Ref. [16] which also included the $\tau$ data and QCD sum rules. Calculations show that another significant improvement (by a factor of about 1.5 ) is possible when high precision $\tau$ lepton decay data are also used. However, for their reliable use one should first understand the reason of the observed discrepancy between two data sets: the two pion spectral functions obtained from $e^{+} e^{-}$and $\tau$ lepton data do not agree confirming earlier evidence for this effect [17]. The solution of this problem should also involve a thorough investigation of the effects of isospin breaking corrections as well as additional radiative effects in $\tau$ decays [18].

The real uncertainty of the theoretical estimate will also strongly depend on the understanding of the role of higher-order effects. These include diagrams with two loops which can be reliably calculated from the $e^{+} e^{-}$data using dispersion relations [19] and much more complicated effects due to hadronic light-by-light scattering. At the present high level of accuracy, both effects give a quite substantial contribution of the order of $100 \cdot 10^{-11}$ each, i.e. about or even larger than the current uncertainty of the leading order hadronic contribution. Recent reevaluations of the pseudoscalar pole contribution dominating in the hadronic light-by-light scattering diagrams revealed that its sign was previously wrong [20]. This initiates new calculations of the higher-order effects and more realistic estimates of their accuracy [21]. 


\section{Summary}

The hadronic effects become an unavoidable part of theory of "pure" QED quantities, such as the anomalous magnetic moment of muon and hyperfine interval in the ground state of muonium. In the case of muon g-2 they limit present accuracy of the theoretical prediction whereas for muonium their impact is more relevant for the future experiments with intensive muon sources and thus higher expected accuracy.

The new experimental result on the anomalous magnetic moment of muon has recently appeared [22]. It has about the same accuracy as theory values from $e^{+} e^{-}$and/or $\tau$ data. A more accurate result is expected soon. The hadronic contributions dominate in the theoretical uncertainty and require a more detailed study. On the contrary, no results on muonium hyperfine structure are expected in near future and theory is more accurate than the experiment. However, to clearly understand what level of accuracy could be a target of new experiments on muonium, one should study theoretical uncertainties. The bound state QED theory is overviewed in Ref. [5] and some progress for $a b$ initio calculations is possible. Better understanding of hadronic effects is needed to find a level of accuracy which cannot be superseded by the ab initio QED calculation. Several projects on the calculation of hadronic effects in physics of muon and muonium which are shortly described here are in progress and we hope to report on their results soon.

\section{Acknowledgements}

The authors would like to thank Andrzej Czarnecki, Michel Davier, Andreas H'ocker, Fred Jegerlehner, Klaus Jungmann and Andrea Vacchi for stimulating discussions. This work was supported in part by the RFBR grant 00-02-16718.

\section{References}

1. S. Geer, Phys. Rev. D 57. 6989 (1998); D 59, 039903 (E) (1998);

B. Autin, A. Blondel and J. Ellis (eds.), Prospective study of muon storage rings at CERN, Report CERN-99-02.

2. S. G. Karshenboim and V. A. Shelyuto, Phys. Lett. B 517, 32 (2001).

3. J. R. Sapirstein, E. A. Terray, and D. R. Yennie, Phys. Rev. D 29, 2290 (1984).

4. A. Karimkhodzhaev and R. N. Faustov, Yad. Fiz. 53, 1012 (1991) (in Russian) (1991); Sov. J. Nucl. Phys. 53, 626 (1991),

R. N. Faustov, A. Karimkhodzhaev and A. P. Martynenko, Phys. Rev. A 59, 2498 (1999).

5. A. Czarnecki, S. I. Eidelman and S. G. Karshenboim, Phys. Rev. D65, 053004 (2002).

6. S. Narison, hep-ph/0108065.

7. R. R. Akhmetshin et al., Phys. Lett. B 527, 161 (2002).

8. R. R. Akhmetshin et al., Phys. Lett. B 466, 385 (1999); Erratum-ibid. B 508, 217 (2001);

M. N. Achasov et al., Phys. Rev. D 63, 072002 (2001).

9. R. N. Faustov and A. P. Martynenko, Phys. Lett. B 541, 135 (2002).

10. S. G. Karshenboim and V. A. Shelyuto, in progress.

11. H. N. Brown et al., Phys. Rev. Lett. 86, 2227 (2001).

12. S. Narison, Phys. Lett. B 513, 53 (2001), Erratum-ibid. B 526, 414 (2002);

J. F. Trocóniz and F. J. Ynduráin, Phys. Rev. D 65, 093001 (2002).

13. S. Eidelman and F. Jegerlehner, Z. Phys. C67, 585 (1995).

14. M. Davier, S. Eidelman, A. Höcker and Z. Q. Zhang, eprint: hep-ph/0208177.

15. J. Z. Bai et al., Phys. Rev. Lett. 88, 101802 (2002).

16. M. Davier and A. Höcker, Phys. Lett. B 435, 427 (1998).

17. S. Anderson et al., Phys. Rev. D 61, 112002 (2000);

S. Eidelman, Nucl. Phys. B (Proc. Suppl.) 98, 201 (2001). 
18. K. Melnikov, Int. J. Mod. Phys., A 16, 4591 (2001);

H. Czyż and J. H. Kühn, Eur. Phys. J. C 18, 497 (2001);

V. Cirigliano, G. Ecker and H. Neufeld, Phys. Lett. B 513, 361 (2001) and hep-ph/0207310.

19. B. Krause, Phys. Lett. B 390, 392 (1997).

20. M. Knecht and A. Nyffeler, Phys. Rev. D 65, 073034 (2002);

M. Hayakawa and T. Kinoshita, hep-ph/0112102;

J. Bijnens, E. Pallante and J. Prades, Nucl. Phys. B 626, 410 (2002);

I. Blockland, A. Czarnecki and K. Melnikov, Phys. Rev. Lett. 88, 071803 (2002).

21. M. J. Ramsey-Musolf and M. B. Wise, Phys. Rev. Lett. 89, 041601 (2002).

22. G. W. Bennett et al., Phys. Rev. Lett. 89, 101804 (2002);

$\mathrm{S}$. Redin et al., this issue. 